\title{
An Interpretive Review: Diet in Early Life and the Prevention of Atherosclerosis
}

\author{
LOIS D. MCBEAN(62) AND ELWOOD W. SPECKMANN
}

National Dairy Council, Division of Nutrition Research, Chicago, Illinois, USA

\begin{abstract}
Atherosclerosis has reached epidemic proportions in the United States (50). Although there are many risk factors which may influence the eventuality of this vascular disease, emphasis has been placed on the composition of dietary lipids $(2,6,7,37,41,46,49,50)$ and genetics $(10,15-17,21-24$, $30,35,39,53,55)$. The etiology of atherosclerosis may be pediatric in origin, as arterial fatty streaks, possibly antecedents of atherosclerotic lesions, have been found in infants 3-4 months of age $(32,33,48,58)$. Thus, dietary intervention, specifically, alteration of dietary fat intake commencing in infancy, has been proposed as a means of primary prevention of atherosclerosis $(2,41,50)$. Several authorities $(1,7,15,16$, $40,46,47,55$ ), however, do not recommend early dietary manipulation for the general population. Potential detrimental consequences of such dietary changes have been hypothesized $(8,12,14,18,29,40,47,53,56,61)$. It is the purpose of this interpretive review to discuss the following: opposing recommendations regarding dietary manipulation in early life as a preventive measure of atherosclerosis; the pathogenesis of atherosclerosis; factors other than diet such as genetics which may be associated with the development of atherosclerosis; possible detrimental effects of early dietary intervention; the circumstance(s) under which dietary changes are justified; and the role of the pediatrician in the prevention of atherosclerosis.
\end{abstract}

\section{RECOMMENDATIONS REGARDING EARLY DIETARY INTERVENTION}

\section{ATHEROSCLEROSIS STUDY GROUP AND EPIDEMIOLOGY STUDY GROUP OF INTER-SOCIETY COMMISSION FOR} HEART DISEASE RESOURCES

Based on the statistical association of dietary composition and the development of atherosclerosis in some but not all population groups, the Atherosclerosis Study Group and Epidemiology Study Group (2) have proposed the following recommendations for the general public from infancy through adulthood: (1) "caloric intake be adjusted to achieve and maintain optimal weight," (2) "reduction of dietary cholesterol to less than $300 \mathrm{mg}$ per day," and (3) "substantial reduction of dietary saturated fats." Dietary fat should contribute less than $35 \%$ of total calories and fat calories should be equally distributed among saturated, monounsaturated, and polyunsaturated sources. The Atherosclerosis Study Group and Epidemiology Study Group stated that "with these dietary principles, requirements for optimal nutrition can be met for all sectors of the population, including infants, children, adolescents, pregnant and lactating women, and older persons." In a position paper prepared by the American Health Foundation (50) it is stated that "the "average American diet' which is relatively high in calories, saturated fat, cholesterol, simple sugars and salt is not optimal for the sedentary way of life of Western man. Dietary modifications, similar in principle to those used in the intervention studies, are capable of ameliorating, reversing or preventing the risk factors of hyperlipidemia, obesity, and possibly hypertension. The programs used in these studies have resulted in an appreciable reduction in incidence of coronary heart disease." In accordance with the above recommendations, Malmros (41) has championed similar changes in diet for the entire population, including children and adolescents. Thus it is emphasized that diet throughout the life of an individual, from infancy through adulthood, be lowered in saturated fatty acids and cholesterol and increased in polyunsaturated fatty acids in an attempt to prevent the development of elevated blood cholesterol levels (hypercholesterolemia) and hence atherosclerosis. Other investigators $(6,37)$ have shown that in certain selected segments of the population dietary changes in cholesterol and/or saturated fatty acids have apparently decreased the incidence of heart disease. Dayton et al. (6) and Leren (37) have reported that a cholesterol-lowering diet fed to older men resulted in a decreased occurrence of myocardial infarction.

\section{COMMITTEE ON NUTRITION OF AMERICAN ACADEMY OF PEDIATRICS}

This committee (1), commenting on the recommendations of the Atherosclerosis Study Group and Epidemiology Study Group (2), stressed that dietary intervention is "at present, experimental and recommends against dietary changes for all children."

\section{SUBCOMMITTEE ON ATHEROSCLEROSIS, COUNCIL OF RHEUMATIC FEVER AND CONGENITAL HEART} DISEASE OF AMERICAN HEART ASSOCIATION

This subcommittee (47), following a 2.5 year study of the problem, concluded that "there is no scientific justification at this time for recommending to the population at large that diets of all children be radically altered in the hope of preventing premature heart disease."

\section{FOOD AND NUTRITION BOARD, NATIONAL ACADEMY OF SCIENCES-NATIONAL RESEARCH COUNCIL, AND COUNCIL ON FOODS AND NUTRITION, AMERICAN MEDICAL ASSOCIATION}

In a joint statement on diet and coronary disease prepared by the above organizations, it is stated that although several risk factors associated with coronary heart disease have been identified, "the evidence is not sufficient to quantitate the benefits that may be expected to come from modifying these various risk factors" (7). It is recommended that a plasma lipid profile be obtained in early adulthood and repeated thereafter at appropriate intervals. Individuals falling into risk categories 
as determined by their levels of lipid in plasma should receive appropriate dietary advice without compromising the intake of essential nutrients (7).

\section{COMMENTS OF VARIOUS INVESTIGATORS}

Schubert (55) has reviewed available data on dietary manipulations for the general population and has concluded that "since the benefits of large scale dietary alterations are unproved and the risks unknown, screening and prospective treatment of the estimated 5 to 7 percent of persons at risk is advocated rather than tampering with the diet of all American children."

Fredrickson $(15,16)$, commenting on the recommendations of the Atherosclerosis Study Group and Epidemiology Study Group of the Inter-Society Commission for Heart Disease Resources (2) posed several questions. "Are we convinced of the safety of a diet containing $10 \%$ of polyunsaturates to the extent that we want to insist on this in baby's formula?" (15) and "is it possible, for example, that enforcement of a nutritional pattern different from breast feeding will alter adaptability to the subsequent diet?" (16), and, furthermore, "what are the possible consequences of greatly increasing the intake of polyunsaturates in childhood? And of continuing this for the remainder of one's life?" (16). It is not known whether the current feeding practices are ideal, but there is no longitudinal evidence to recommend national dietary changes (55).

Meade and Chakrabarti (46) state that a causal relation between childhood diet and adult heart disease has yet to be confirmed by reliable scientific evidence and also, that restriction of dietary cholesterol and/or saturated fatty acids in the population at large has not been shown to decrease the incidence of coronary heart disease (CHD). They further claim that the "prime need---is still, and will probably long remain, the identification of causes and mechanisms whose subsequent modification will lead to large rather than marginal improvements in incidence." "Intervention studies are, with few exceptions, not at present likely to improve understanding of aetiology or pathogenesis very substantially. The foreseeable chances of successful primary prevention on any major scale are slender, particularly by the modification of personal habits. There is a pressing need for prospective observational studies in which new risk factors are identified, particularly those likely to give a more direct measure of thrombotic tendency."

\section{DISCUSSION}

\section{PATHOGENESIS OF ATHEROSCLEROSIS}

It has been proposed, although not proven, that pathologically atherosclerosis progresses in a series of definite steps, namely: (1) the occurrence of arterial fatty streaks in infancy and childhood, (2) the formation of a fibrous plaque in the 2 nd decade of life, (3) complicated lesions evident in the 4 th and later decades, and (4) the episodes of clinical manifestations, such as heart disease, in later life $(32,34,39,42-44$, $48,58,59$ ).

Fatty streaks are evident in infants by $3-5$ months of age and remain as such for the first 2 decades of life $(32,34,44$, 48). The incidence of fatty streaks appears to be quite consistent regardless of the considerable geographic differences in the mortality rate from CHD. A group of cooperating pathologists involved in the International Atherosclerosis Project (IAP) examined 23,000 sets of coronary arteries and aortas from autopsied persons in 14 countries and found no consistent differences in aortic fatty streaks among groups from different geographic locations (43). However, great individual differences exist in fatty streaking between two persons of the same age, sex, and geographic area. As a child ages it is assumed that the number and size of fatty streaks increase. It is possible that some deposits are resorbed, while others progress to fibrous plaques. A number of questions remain unanswered. What is the significance of fatty streaks in the genesis and location of plaques? Can the process be reversed at the fatty streak stage? Is it possible to slow down the progression of fatty streaks to fibrous plaques? What factors initiate the process and influence the progression (34)? There is a difference of opinion among authorities concerning the question of whether fatty streaks are a precedent of CHD in adulthood. McMillan (44) considers fatty streaks to be a locus for the development of plaques, yet believes that fatty streaks are unrelated to heart disease.

Plaques are believed to result from the accumulation of lipid in fatty streaks. The arterial wall also reacts to certain lipids by proliferation of connective tissue elements. The result is a fibrous plaque with fat concentrated at the base covered by a cap of connective tissue. Fibrous plaques have been observed in men in their 20's killed during the Korean and Vietnam wars $(9,45)$. McNamara et al. (45) observed a lesser incidence $(45 \%)$ of coronary atherosclerosis in the Vietnam casualties than in Korean victims $(77.3 \%)$.

Enos et al. (9) view stress on the intima which results from the hemodynamics of coronary circulation as responsible for plaque formation. This theory implies a genetic influence due to anatomic variations in the coronary vascular system. Elasticity of the vascular system can also vary with age. Plaques are not identical but rather are of varied compositions (44). They also seem to exist in specific locations within the artery, which tends to support the role of blood flow and pressure in their genesis. It is therefore concluded that plaques do not comprise a precise disease entity and that they probably develop via dissimilar etiologic and pathogenetic mechanisms. The initial stages of plaque formation have not been elucidated.

Complicated lesions in the 4th and later decades of life are the result of additional changes in fibrous plaques. Data from the IAP (43) revealed that the extent of advanced lesions in both aorta and coronary arteries varied among population groups and that the development of complicated lesions was more advanced in populations with a greater incidence of CHD than in those populations with a lesser incidence. Clinical episodes such as myocardial infarction (32) constitute the fourth stage. The first three stages, namely: fatty streak, fibrous plaque, and complicated lesions, occur in the arterial wall before the manifestation of clinical disease. Different factors may be responsible for the various stages of atherosclerotic lesions and still other factors may influence the clinical occurrence of the disease.

\section{FACTORS OTHER THAN DIET ASSOCXATED WITH COURSE OF ATHEROSCLEROSIS}

A multitude of interrelated factors, such as genetics, hypercholesterolemia, smoking, elevated blood pressure (hypertension), diabetes, physical inactivity, and stress, are believed to be associated with the development of atherosclerosis and subsequent involvement of vital organs $(2,7,11$, $34,40,46,47)$. Other factors still remain to be identified. A combination of the above factors, rather than each occurring singly, increases risk (2). Genetic influences and blood lipid disorders have received the most attention therefore these two identifiable risk factors will be discussed.

The relative role of genetic factors in the etiology of atherosclerosis is less controversial than the role of diet $(15-17,21-24,30,35,38,40,47,57)$. A series of genetic lipoprotein disorders associated with precocious atherosclerosis and diagnosed by an estimation of serum cholesterol and triglyceride levels has recently been identified $(11,15-17$, $38)$. Fredrickson $(15-17)$ has described the existence of five (type $I$ through type $V$ ) genetically determined causes of 
hyperlipidemia, all of which require type-specific dietary modifications. Fat-induced hypertriglyceridemia (type $I$ ) is rare and is not a precursor of atherosclerotic disease $(38,39)$, whereas types II, III, IV, and possibly $V$ are related to premature atherosclerosis (17). Type II is the commonest type of primary hyperlipidemia (familial hypercholesterolemia) seen in childhood and it can be detected in infancy by elevated cord blood total and $\beta$-lipoprotein cholesterol $(19,35,38)$. The incidence of type II is 1 out of every 200 infants tested. The disease in the homozygous state is associated with premature CHD before age 20 . In the heterozygous state it appears to be associated with premature CHD in the 4th and later decades (39). Type $I I$ is presumably transmitted as an autosomal dominant gene, although the genetic basis of this disorder has not been unequivocally established $(21,22,35$ 38). Kwiterovich et al. (35) concluded from a study with 29 infants with type II hypercholesterolemia that if one parent of each child were known to have the disorder, identification of the child with type II could be made by measurement of the plasma concentration of low density lipoproteins. Types III and $V$ hyperlipidemia are undetected before adulthood (38). Type IV (primary endogenous hypertriglyceridemia) is associated with CHD in early adult life or later and had been reported to be infrequent in childhood (39), with the probability that a more accurate assessment of its prevalence will be obtained through several large pediatric lipid surveys currently underway. Type $I V$ is probably autosomal dominant (21).

Various other investigators $(10,11,23,24,30,57)$ support the genetic origin of hyperlipidemia (both hypertriglyceridemia and hypercholesterolemia). Slack and Evans (57) have provided evidence of an increased risk of ischemic heart disease in relatives of patients with this disease, possibly because of genetic factors. Goldstein et al. (24) investigated the genetics of hyperlipidemia in coronary heart disease. The frequency of monogenic hyperlipidemia in the general population was found to be about $1 \%$ with at least 1 in 160 individuals carrying a gene for familial hypercholesterolemia, familial hypertriglyceridemia, or familial combined hyperlipidemia. Thus the public health problems associated with this gene frequency are of major proportions. Epstein (10) and Epstein and Ostrander (11) have studied the possible role of genetic influences in predisposing individuals or groups to the complications of atherosclerosis. Family history as a predictive element of heart disease for individuals was found more important when death from heart disease occurred in early life than in later years. Clarkson et al. (5) have demonstrated the influence of genetics on blood lipid levels in squirrel monkeys; $92 \%$ of the variability in plasma cholesterol concentrations in the squirrel monkeys was the result of genetic influences. Hazzard et al. (30) studied the relation between lipoprotein phenotypes (Fredrickson classification) and genetic lipid disorders in 156 genetically defined survivors of myocardial infarction. It was concluded that on an individual basis no lipoprotein pattern proved to be specific for any particular genetic lipid disorder and conversely no genetic disorder was specified by a single lipoprotein pattern. This study indicates that lipoprotein phenotypes are not reliable genetic markers, as individuals can vary in lipoprotein phenotype with time.

Of all the lipid and lipid-bearing substances which are found in blood and have been incriminated in atherogenesis, emphasis has been on cholesterol and triglycerides $(3,4,17$, $23,41,54)$. Both hypercholesterolemia and hypertriglyceridemia are considered to be predisposing risk factors, independent of each other, for atherosclerosis (4). An estimation of both serum cholesterol and triglyceride levels is necessary in order to identify persons with ischemic heart disease $(4,23$, 54). It has been unequivocally demonstrated that dietary lipids dramatically affect serum lipids and lipoproteins $(28,51)$. Dietary habits in terms of the type and amount of nutrients, in addition to total caloric intake, are generally developed in the 1 st and 2 nd decades of life; consequently, this period in the life cycle may be the most appropriate time to establish sound eating habits (42). Some investigators are of the opinion that most serum lipid disorders originate in childhood, suggesting a strong implication for the role of genetics (34). Others $(26,27$, 59 ) believe that diet is relatively unimportant in the genesis of fatty streaks in infancy and childhood, yet may be of importance in the formation of fibrous plaques in the 2 nd decade of life. It has not been established whether lipid analysis of blood during infancy and childhood serves as an accurate predictor of adult blood lipid values (34).

Infants fed fat from either human or cow's milk have higher serum cholesterol concentrations than those infants receiving proprietary infant formulas which are comprised of a mixture of corn and coconut oils or of soy oil (13); however, the serum cholesterol level for optimal physiologic function and growth during the 1st year of life is unknown (55). The majority of infants in the United States consume commercially-prepared formulas which have more polyunsaturated fatty acids, and consequently these infants have lower serum cholesterol concentrations than do breast-fed infants $(12,40)$. A study concerning the long range consequences on development and health of consuming a diet high in polyunsaturated fatty acids needs to be undertaken.

The effect of cholesterol intake on the development of atherosclerotic lesions is not firmly established. Pickering et al. (49) found that feeding monkeys a formula containing fat mainly of vegetable oil origin resulted in significantly lower levels of serum cholesterol compared with the levels found in animals receiving a milk fat formula; however, the histologic features of the cardiovascular system were similar for both groups. Greenberg and Gonzalez (26) and Greenberg and Wheeler (27) fed infant monkeys one of the following four diets: (1) monkey milk, (2) human milk, (3) bovine butterfat formula, and (4) a mixed fat formula with a polyunsaturated to saturated fatty acid ratio of $1 / 1$. The monkeys fed the bovine milk fat had significantly elevated total plasma cholesterol concentrations; however, there was no association between the frequency and severity of arterial lesions and the blood cholesterol levels. Similarly, the presence of high levels of polyunsaturated fatty acids in the diet was not correlated with a lesser incidence of vascular lesions. The IAP (43) found that coronary lesions were related to serum cholesterol and dietary fat only when comparing populations, not individuals, a fact supporting the findings of Greenberg et al. $(26,27)$.

No one has demonstrated that the modification of dietary lipids as suggested by the Atherosclerosis Study Group and Epidemiology Study Group (2) prevents, mitigates, or cures coronary vascular disease or improves the overall lifespan (52).

\section{POSSIBLE DETRIMENTAL EFFECTS OF EARLY DIETARY INTERVENTION}

Several investigators $(1,8,12,14,18,29,40,47,53,56$, $61)$ have speculated on the potential hazards of the widespread feeding practices and recommendations that result in continued low serum cholesterol levels. It has been proposed that an intake of dietary cholesterol early in life may initiate enzyme systems responsible for serum cholesterol degradation in adulthood $(12,29,53)$. Reiser and Sidelman (53), Sidelman (56), and Hahn and Kirby (29) investigated the problem in rats to establish whether high intakes of cholesterol in newborn and suckling rats would serve to stabilize cholesterol anabolic, catabolic, and/or transport processes so that in adulthood serum cholesterol homeostasis could be maintained at low levels during cholesterol ingestion. The adult male rats, but not females, when fed cholesterol as infants, had lower serum cholesterol concentrations than adult male rats who had received little or no cholesterol during infancy (53). Thus cholesterol intake during the suckling period may, by an 
unknown mechanism, establish a precedent, at least in the adult rat, for maintainance of low serum cholesterol concentration in adulthood regardless of high cholesterol intakes. Glueck et al. (20) investigated variations in the intake of dietary cholesterol and polyunsaturated or saturated fatty acids in infants during the 1 st year of life to establish whether the intake of dietary cholesterol in early infancy determines subsequent adaptation to increased dietary cholesterol in later infancy and childhood. The plasma cholesterol concentrations in the infants at 12 months of age did not appear to be affected by antecedent low or moderate cholesterol ingestion. Thus the results of Glueck et al. (20) with human infants did not support the findings of Reiser and Sidelman (53), Sidelman (56), and Hahn and Kirby (29). However, the lack of agreement in the results between the human and animal data may perhaps be explained by differences in experimental design such as the timing and the duration of the suckling period and the lifespan. It may be that the effects of the low cholesterol diets in the infants in the study conducted by Glueck et al. (20) will be expressed much later in the life of the child, rather than 5-6 months after the dietary change.

Fomon (12) speculated, without offering evidence, that the rate of myelination in the brain and nervous system may be adversely affected by an intake of cholesterol-free diets and by diets low in long chain fatty acids. Fumagalli et al. (18) reported that rats fed drugs inhibiting the biosynthesis of cholesterol had retarded rates of brain myelination. It has been stated that "it is possible that diets based on vegetable oils may interfere with myelination of the brain because they do not provide cholesterol as such and because the polyunsaturated fatty acids present in such diets alter the quantity and type of fats that circulate in the body" (12). Widdowson (61) found high calcium excretion in infants fed formulas in which butterfat had been replaced by an animal-vegetable oil mixture. The oleo oil (destearinated beef fat) produced poor calcium absorption.

Another consequence of consuming a diet low in cholesterol and saturated fatty acids and high in unsaturated fatty acids may be the formation of gallstones $(31,60)$. Sturdevant $e t$ al. (60) reported from autopsy data that $34 \%$ of men consuming an experimental diet in which vegetable oils were substituted for about two-thirds of the animal fats developed gallstones. In contrast, $14 \%$ of the control subjects who received a conventional diet containing $40 \%$ fat calories, principally of animal origin, had gallstones. The authors thus concluded that "the data suggest that some property of the experimental diet (high ratio of polyunsaturated fat to saturated fat or high ratio of plant sterol to cholesterol or both) promoted gallstone formation."

Skim milk, in preference to whole milk, has frequently been fed to infants beginning at 4-6 months of age to prevent atherosclerosis (14). Fomon (14) suggests that "when skim milk serves as a major source of calories during infancy, total calorie intake and intake of fat are likely to be undesirably low and intakes of protein and lactose are likely to be undesirably high." Based on the results of several studies, Fomon (14) recommends that skim milk, although a reasonable food for children beyond infancy, is not satisfactory as a food for infants.

\section{JUSTIFICATION FOR DIETARY INTERVENTION AND ROLE OF PEDIATRICIAN}

Dietary intervention early in life has been prescribed for children with type II hyperlipidemia, a genetic disorder (17, $19,20,36,55)$. The goal is to reduce plasma low density lipoprotein cholesterol concentration; therefore, a therapeutic diet low in cholesterol with a polyunsaturated to saturated fatty acid ratio of $2 / 1$ is recommended. Saturated fat should not exceed $10 \%$ and total fat $35 \%$ of total food calories.
Glueck and Tsang (19) studied the effect of diet on plasma lipids during the 1st year of life in infants with one type of familial type II hyperlipoproteinemia. Although success was attained in the early specific identification of type II via cord blood samples and dietary manipulation, the investigators expressed concern regarding the existence of any potentially harmful side effects of feeding low cholesterol, polyunsaturate-rich diets to infants. Serum cholesterol concentration in infancy can be drastically altered; however, the consequences of a low cholesterol, high polyunsaturate diet, even in those infants and children with genetic abnormalities, are unknown. Long term studies need to be conducted to determine the efficacy or lack of efficacy of dietary regimens in the prevention of atherosclerosis.

Heredity may be the final link in the chain of preventive cardiology; therefore, every effort should be made to identify infants and children with a familial pedigree of serious atherosclerosis. Serum lipid analysis early in life (even at birth) will frequently detect this metabolic disorder. Any infant born into a family with a known history of familial hypercholesterolemia should be tested. Early identification of hyperlipidemia may avert the premature onset of ischemic heart disease and other forms of atherosclerosis. Type II hyperlipoproteinemia is an ideal condition to test the hypothesis that a decrease in plasma lipid levels prevents or delays the atherosclerotic process (38). The pediatrician can become involved in the prevention of cardiovascular disease by becoming interested in the development and utilization of screening techniques for the early detection of risk factors (25). Although the need for stringent atherosclerosis prophylaxis in early life is probably largely for the minority, those at risk of developing CHD should be identified. Once the total risk profile is established, then individual treatment such as diet therapy, family counseling, and behavior modification can be employed to decrease those factors which elevate total risk. It is hoped that the pediatrician with the generalist, internist, and cardiologist will become involved in the intervention of cardiovascular disease.

\section{SUMMARY}

The presence of arterial fatty streaks has been observed in infants by $3-5$ months of age, thus suggesting that pathologically, atherosclerosis may have its origin in infancy. A number of factors have been incriminated as influencing the development of atherosclerosis; however, emphasis has been placed on dietary lipid composition and genetics. Although conclusive evidence proving a causative relation between the composition of the diet, the development of atherosclerotic lesions, and the subsequent onset of atherosclerosis in an individual is lacking, dietary recommendations for the general population, from infancy through adulthood, have been posed. Such dietary recommendations involve a decreased consumption of cholesterol and saturated fatty acids and an increased intake of polyunsaturated fatty acids.

The lack of currently available data has created a difference of opinion regarding the efficacy or lack of efficacy of dietary modifications on the rates of premature atherosclerotic disease. Some investigators claim that dietary manipulations that result in decreased dietary cholesterol and saturated fatty acids may be detrimental. Dietary cholesterol in early life may be essential for the myelination of the brain as well as important for the regulation of cholesterol in later life. Long term studies to justify dietary modification for the general population are needed. At present, it appears advantageous to identify and treat in well designed studies the $5-7 \%$ of children in the United States who are genetically predisposed to atherosclerosis. It is emphasized that the pediatrician can have an active role in preventive cardiology by identifying infants and children with genetic lipoprotein disorders and initiating prophylaxis for persons at risk. 


\section{REFERENCES AND NOTES}

1. American Academy of Pediatrics, committee on Nutrition Childhood diet and coronary heart disease. Pediatrics, 49: 305 (1972).

2. Atherosclerosis Study Group and Epidemiology Study Group of the Inter-Society Commission for Heart Disease Resources: Primary prevention of the atherosclerotic diseases. Circulation, 42: A-55 (1970)

3. Brown, D. F., Kinch, S. H., and Doyle, J. T.: Serum triglycerides in health and in ischemic heart disease. N. Engl. J. Med., 273: 947 (1965).

4. Carlson, L. A., and Bottiger, L. E.: Ischaemic heart-disease in relation to fasting values of plasma triglycerides and cholesterol. Lancet, $i$ : 865 (1972).

5. Clarkson, T. B., Lofland, H. B., Jr., Bullock, B. C., and Goodman, H. O.: Genetic control of plasma cholesterol. Arch. Pathol., 92: 37 (1971).

6. Dayton, S., Chapman, J. M., Pearce, M. L., and Popjak, G. J. Cholesterol, atherosclerosis, ischemic heart disease, and stroke. Ann. Intern. Med., 72: 97 (1970)

7. Diet and coronary heart disease. A joint statement of the Food and Nutrition Board, Divison of Biology and Agriculture, National Academy of Sciences-National Research Council, and The Council on Foods and Nutrition, American Medical Association, 1972 .

8. Egwim, P. O., and Sgoutas, D. S.: The fatty acids of adrenal lipids from rats fed partially hydrogenated soybean fat. J. Nutr., 101 315 (1971).

9. Enos, W. F., Jr., Beyer, J. C., and Holmes, R. H.: Pathogenesis of coronary disease in American soldiers killed in Korea. J. Amer. Med. Ass., 158: 912 (1955)

10. Epstein, F. H.: Risk factors in coronary heart disease environmental and hereditary influences. Isr. J. Med. Sci., 3: 594 (1967).

11. Epstein, F. H., and Ostrander, L. D., Jr.: Detection of individual susceptibility toward coronary disease. Prog. Cardiovasc. Dis. 13: 324 (1971).

12. Fomon, S. J.: A pediatrician looks at early nutrition. Bull. N. Y. Acad. Med., 47: 569 (1971)

13. Fomon, S. J.: Infant Nutrition (W.B. Saunders Co., Philadelphia 1967).

14. Fomon, S. J.: Skim milk in infant feeding. Commentaries on Infan and Child Nutrition, Maternal and Child Health Service, January 1973

15. Fredrickson, D. S.: Mutants, hyperlipoproteinaemia, and coronary artery disease. Brit. Med. J., 2: 187 (1971).

16. Fredrickson, D. S.: Symposium: Factors in childhood that influence the development of atherosclerosis and hypertension Introduction. Amer. J. Clin. Nutr., 25: 221 (1972)

17. Fredrickson, D. S., and Levy, R. I.: Familial hyperlipoproteinemia In: J. B. Stanbury, J. B. Wyngaarden, and D. S. Fredrickson: The Metabolic Basis of Inherited Disease (McGraw-Hill Book Co., Inc., New York, 1972).

18. Fumagalli, R., Smith, M. E., Urna, G., and Paoletti, R.: The effect of hypocholesteremic agents on myelinogenesis. J. Neurochem. 16: 1329 (1969)

19. Glueck, C. J., and Tsang, R. C.: Pediatric familial type II hyperlipoproteinemia: effects of diet on plasma cholesterol in the first year of life. Amer. J. Clin. Nutr., 25: 224 (1972).

20. Glueck, C. J., Tsang, R., Balistreri, W., and Fallat, R.: Plasma and dietary cholesterol in infancy: Effects of early low or moderate dietary cholesterol intake on subsequent response to increased dietary cholesterol. Metabolism, 21: 1181 (1972).

21. Glueck, C. J., Tsang, R., Fallat, R., Buncher, C. R., Evans, G., and Steiner, P.: Familial hypertriglyceridemia: Studies in 130 children and 45 siblings of 36 index cases. Metabolism, 22: 1287 (1973).

22. Glueck, C. J., Fallat, R., Buncher, C. R., Tsang, R., and Steiner, P. Familial combined hyperlipoproteinemia: Studies in 91 adults and 95 children from 33 kindreds. Metabolism, 22: 1403 (1973).

23. Goldstein, J. L., Hazzard, W. R., Schrott, H. G., Bierman, E. L. and Motulsky, A. G.: Hyperlipidemia in coronary heart disease. I. Lipid levels in 500 survivors of myocardial infarction. J. Clin. Invest., 52: 1533 (1973).

24. Goldstein, J. L., Schrott, H. G., Hazzard, W. R., Bierman, E. L., and Motulsky, A. G.: Hyperlipidemia in coronary heart disease. II. Genetic analysis of lipid levels in 176 families and delineation of a new inherited disorder, combined hyperlipidemia. J. Clin. Invest., 52: 1544 (1973)

25. Graham, M. F.: Prevention of cardiovascular disease. J. Amer. Med. Ass., 224: 1189 (1973),

26. Greenberg, L. D., and Gonzalez, I. E.: Influence of fatty acid composition of infant formulas on development of arteriosclerosis. Exp. Mol. Pathol., 10: 240 (1969).

27. Greenberg, L. D., and Wheeler, P.: Influence of fatty acid composition of infant formulas on the development of arteriosclerosis and on the lipid composition of blood and tissues. I Lipid studies. In: M. F. Brink, and D. Kritchevsky: Symposium
Dairy Lipids and Lipid Metabolism (Avi Publishing Co., Inc., Westport, Conn, 1968)

28. Grundy, S. M., and Ahrens, E. H., Jr.: The effects of unsaturated fats on absorption, excretion, synthesis, and distribution of cholesterol in man. J. Clin. Invest., 49: 1135 (1970).

29. Hahn, P., and Kirby, L.: Immediate and late effects of premature weaning and of feeding a high fat or high carbohydrate diet to weanling rats. J. Nutr., 103: 690 (1973).

30. Hazzard, W. R., Goldstein, J. L., Schrott, H. G., Motulsky, A. G., and Bierman, E. L.: Hyperlipidemia in coronary heart disease. III. Evaluation of lipoprotein phenotypes of 156 genetically defined survivors of myocardial infarction. J. Clin. Invest., 52 : 1569 (1973).

31. Hofmann, A. F., Northfield, T. C., and Thistle, J. L.: Can a cholesterol-lowering diet cause gallstones? N. Engl. J. Med., 288: 46 (1973).

32. Holman, R. L.: Atherosclerosis-a pediatric nutrition problem? Amer. J. Clin. Nutr., 9: 565 (1961)

33. Holman, R. L., McGill, H. C., Jr., Strong, J. P., and Geer, J. C.: The natural history of atherosclerosis. The early aortic lesions as seen in New Orleans in the middle of the 20th century. Amer. J. Pathol., 34: 209 (1958).

34. Kannel, W. B, and Dawber, T. R.: Atherosclerosis as a pediatric problem. J. Pediat., 80: 544 (1972).

35. Kwiterovich, P. O., Jr., Levy, R. I., and Fredrickson, D. S.: Neonatal diagnosis of familial type-II hyperlipoproteinaemia. Lancet, $i$ : 118 (1973)

36. Lees, R. S., and Wilson, D. E.: The treatment of hyperlipidemia. N. Engl. J. Med., 284: 186 (1971).

37. Leren, P.: The Oslo diet-heart study: eleven-year report. Circulation, 42: 935 (1970).

38. Levy, R. I., and Rifkind, B. M.: Diagnosis and management of hyperlipoproteinemia in infants and children. Amer. J. Cardiol., 31: 547 (1973)

39. Lloyd, J. K., and Wolff, O. H.: A paediatric approach to the prevention of atherosclerosis. J. Atheroscler. Res., 10: 135 (1969).

40 Lowe, U. Research in infant nutrition: The untapped well. Amer. J. Clin. Nutr., 25: 245 (1972).

41. Malmros, H.: Dietary prevention of atherosclerosis. Lancet, ii: 479 (1969).

42. McGandy, R. B.: Adolescence and the onset of atherosclerosis. Bull. N. Y. Acad. Med., 47: 590 (1971)

43. McGill, H. C., Jr. (Ed.): The Geographic Pathology of Atherosclerosis (The Williams \& Wilkins Co., Baltimore, 1968).

44. McMillan, G. C.: The onset of plaque formation in arteriosclerosis. Acta Cardiol. 11(Suppl.): 43 (1965).

45. McNamara, J. J., Molot, M. A., Stremple, J. F., and Cutting, R. T. Coronary artery disease in combat casualties in Vietnam. J. Amer. Med Ass, 216:1185 (1971).

46. Meade, T. W., and Chakrabarti, R.: Arterial-disease research: Observation or intervention? Lancet, $i i: 913$ (1972).

47. Mitchell, S., Blount, S. G., Jr., Blumenthal, S., Jesse, M. J., and Weidman, W. H.: The pediatrician and atherosclerosis. Pediatrics, 49: 165 (1972)

48. Moon, H. D.: Coronary arteries in fetuses, infants, and juveniles. Circulation, 16: 263 (1957).

49. Pickering, D. E., Fisher, D. A., Perley, A., Basinger, G. M., and Moon, H. D.: Influence of dietary fatty acids on serum lipids. Amer. J. Dis. Child., 102: 42 (1961).

50. Public Information Subcommittee: Position statement on diet and coronary heart disease. Prev. Med., 1: 255 (1972).

51. Quintao, E., Grundy, S. M., and Ahrens, E. H., Jr.: Effects of dietary cholesterol on the regulation of total body cholesterol in man. J. Lipid Res., 12: 233 (1971).

52. Reiser, R.: Saturated fat in the diet and serum cholesterol concentration: a critical examination of the literature. Amer. J. Clin. Nutr., 26: 524 (1973).

53. Reiser, R., and Sidelman, Z.: Control of serum cholestero homeostasis by cholesterol in the milk of the suckling rat. J. Nutr., 102: 1009 (1972).

54. Rifkind, B. M., Lawson, D., and Gale, M.: Diagnostic value of serum lipids and frequency of lipoprotein patterns in myocardial infarction. J. A theroscler. Res., 8: 167 (1968).

55. Schubert, W. K.: Fat nutrition and diet in childhood. Amer. J. Cardiol., 31: 581 (1973).

56. Sidelman, $Z$. The influence of milk cholesterol ingested by suckling rats on serum cholesterol concentrations of the adult rat (Abstract). Dairy Sci., 34: 820 (1972).

57. Slack, J., and Evans, K. A.: The increased risk of death from ischaemic heart disease in first degree relatives of $121 \mathrm{men}$ and 96 women with ischaemic heart disease. J. Med. Genet., 3: 239 (1966).

58. Strong, J. P., and McGill, H. C., Jr.: The pediatric aspects of atherosclerosis. J. Atheroscler. Res., 9: 251 (1969).

59. Strong, J. P., McGill, H. C., Jr., Tejada, C., and Holman, R. L.: The natural history of at 
lesions in New Orleans, Guatemala, and Costa Rica. Amer. J. Pathol., 34: 731 (1958).

60. Sturdevant, R. A. L., Pearce, M. L., and Dayton, S.: Increased prevalence of cholelithiasis in men ingesting a serum-cholesterol lowering diet. N. Engl. J. Med., 288: 24 (1973).

61. Widdowson, E. M.: The relation between the nature of the fat in

Copyright $\odot 1974$ International Pediatric Research Foundation, Inc. the diet of young babies and their absorption of calcium. Biol. Neonate, 9: 279 (1966).

62. Requests for reprints should be addressed to: L. D. McBean, M.S.

R.D., Nutrition Research Division, National Dairy Council, 111 N. Canal St., Chicago, Ill. 60606 (USA). 